\title{
Centralized Backoff Control Scheme in Slotted CDMA Systems
}

\author{
Intaek Lim, Member, KIMICS
}

\begin{abstract}
This paper proposes a centralized backoff control scheme for guaranteeing fair packet transmissions in S-ALOHA CDMA system. In S-ALOHA CDMA system, simultaneously transmitted packets act as multiple access interferences, so that unsuccessful packet transmissions are caused entirely by multiple access interferences. Therefore, in order to maximize the system performance, the levels of MAI should be kept at an optimal level. In the proposed scheme, the base station broadcasts the packet transmission probability, which is calculated based on the level of MAI. Mobile stations attempt to transmit their packet with the received probability. Simulation results show that the proposed scheme can guarantee a good fairness among all the mobile stations.
\end{abstract}

Index Terms-MAC, Backoff control, CDMA system, Slotted ALOHA

\section{INTRODUCTION}

CDMA technique provides the following advantages: a multiple access capability, a potential for high spectrum efficiency, external interference rejection capability, and inherent frequency diversity [1][2]. This is shown in that most proposals for radio transmission technology (RTT) in IMT-2000 are based on CDMA [3][4].

In S-ALOHA protocol, it is assumed that whenever more than one packet is transmitted at the same time slot, the information in the transmitted packets will be lost. Moreover, if a high traffic load is offered to the system, then the system will become unstable [5]. This would not be the case if it was used with CDMA technique. Application of the conventional S-ALOHA protocol to CDMA technique, namely S-ALOHA CDMA system, offers relatively high system capacity [6][7].

Spreading code protocol in S-ALOHA CDMA system can be classified into shared code and transmitter-based code method [8]. In the shared code method, all the mobile stations share a finite number of spreading codes to communicate with the base station. Whenever a mobile station has a packet to send, it will randomly choose one spreading code and transmit at the next slot. On the other hand, in the transmitter-based code method, a unique spreading code is assigned to each mobile station. It allows

\footnotetext{
Manuscript received December 22, 2010; revised January 18, 2011; accepted January 21, 2010.

Intaek Lim is with the Department of Embedded IT, Pusan University of Foreign Studies, Busan, 608-738, Korea (Email: itlim@,pufs.ac.)
}

several mobile stations to transmit their packet at the same slot using a different spreading code for each one.

In S-ALOHA CDMA system with a transmitter-based code method, if the number of transmitting mobile stations increases above a certain threshold, almost all the packets received by the base station can be erroneous. Hence, unsuccessful packet transmissions are caused entirely by the number of simultaneously transmitting mobile stations if negligible thermal noise effects assumed. If the level of multiple access interferences can be remained close to the level that the system can support, it is expected to achieve the best system performance. Therefore, the number of simultaneously transmitting mobile stations needs to be limited with the use of backoff control scheme.

There are some researches for the backoff control aiming at improving the system throughput [11-14]. In these researches, the mobile station decreases its transmission probability when it fails in transmission. Continuously decreasing the transmission probability, a specific mobile station restricts excessively its transmission. As a result, the system throughput can be degraded, the transmission delay can be increased, and moreover, fairness between mobile stations cannot be guaranteed.

This paper is intended to improve the throughput and delay performance and guarantee the fairness between mobile stations. For these purposes, this paper proposes a backoff control scheme for S-ALOHA CDMA system with a transmitter-based code method. In the proposed scheme, the base station controls the transmission probability of mobile stations based on the traffic loads.

This paper is organized as follows. Section II describes the S-ALOHA CDMA system model. The proposed backoff control scheme is explained in Section III, and simulation results are presented in Section IV. Concluding remarks are presented in Section V.

\section{S-ALOHA CDMA SYSTEM}

\section{A. Research motivations}

In this paper, the bit error probability $P_{e}(m)$ of CDMA system is assumed as follows [10]:

$$
P_{e}(m)=Q\left(\left[\frac{m-1}{3 N}+\frac{N_{0}}{E_{b}}\right]^{-\frac{1}{2}}\right)
$$


Here, $N$ is the processing gain, $m$ is the number of simultaneously transmitted packets, $E_{b} / N_{0}$ is the ratio of energy-per-bit to noise power spectral density, and $Q(x)$ is given by

$$
Q(x)=\frac{1}{\sqrt{2 \pi}} \int_{x}^{\infty} e^{-u^{2} / 2} d u
$$

From (1), the number of simultaneously transmitted packets as well as the processing gain has a strong influence on the bit error probability of CDMA system. Accordingly, the system throughput, which is defined as the number of successful packets, can be affected by the bit error probability. It is assumed that a packet is successfully received at the base station when all the bits of a packet are error-free. When $m$ packets are transmitted simultaneously, the probability $P_{s}(m)$ that a packet is successfully received and the number of successful packets $S(m)$ can be expressed as follows, respectively:

$$
\begin{aligned}
& P_{S}(m)=\left\{1-P_{e}(m)\right\}^{L} \\
& S(m)=m \cdot\left\{1-P_{e}(m)\right\}^{L}
\end{aligned}
$$

where $L$ is the length of a packet in bits.

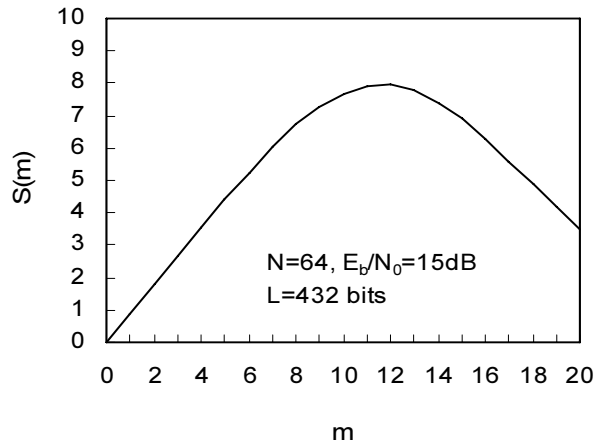

Fig.1. Throughput of S-ALOHA CDMA system.

Fig.1 shows the achievable throughput of CDMA system versus the number of simultaneously transmitted packets, where the packet length is 432 bits, the processing gain is 64 , and $E_{b} / N_{0}$ is $15 \mathrm{~dB}$. As shown in Fig.1, when the number of simultaneously transmitted packets is over 12, the throughput decreases due to the excessive multiple access interferences. Therefore, in order to achieve the maximum throughput, the number of simultaneously transmitted packets should be controlled by the base station.

\section{B. Slotted CDMA system}

Fig.2 shows the S-ALOHA CDMA system model. The system consists of a base station and $K$ mobile stations, each with an infinite buffer capacity. Each packet has a fixed length of $L$ bits, which is equal to slot duration. Mobile stations synchronize their transmissions so that they transmit packets with a uniquely assigned transmitter-based code at the beginning of each slot.
Every mobile station generates a packet in each slot with arrival rate $\lambda$. When the mobile station generates a packet, it is stored at the buffer. Stored packets are served on a first-in-first-out discipline.

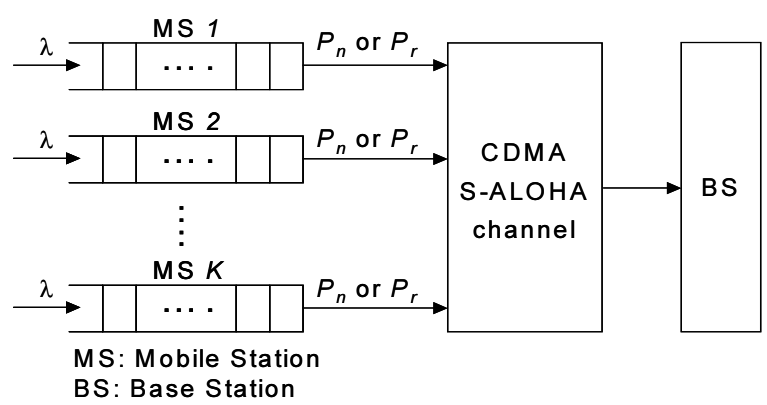

Fig.2. S-ALOHA CDMA system model.

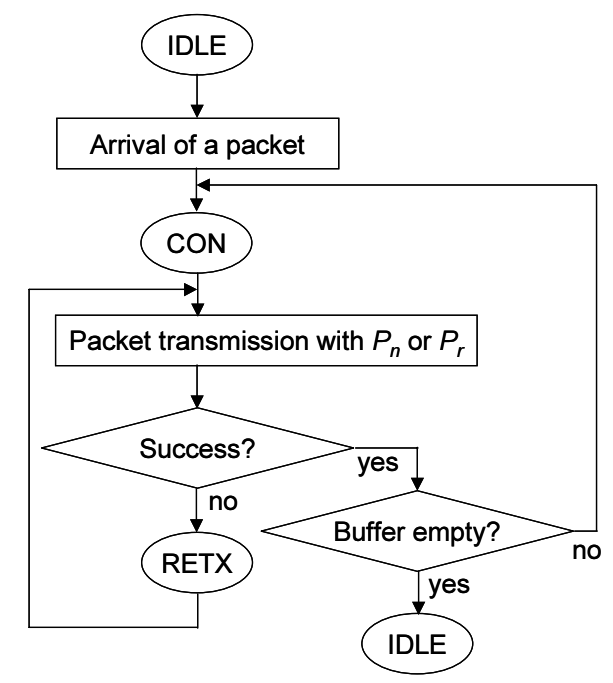

Fig.3. Operation of mobile station.

Fig. 3 shows the flow descriptions for the mobile station. All the mobile stations may be in one of three different operation states: idle state, contention $(\mathrm{CON})$ state, and retransmission (RETX) state. The mobile station, which does not have any packet in the buffer, is said to be in the idle state. When the mobile station in the idle state generates a packet, it enters into the contention state and transmits a packet at the next slot with a given transmission probability $P_{n}$. The mobile stations are informed as to whether or not the transmitted packets are successfully received by the base station in the form of acknowledgement. The mobile station that experiences a packet error or does not permitted to transmit enters into the retransmission state, and retransmits it at the next slot with a given retransmission probability $P_{r}$. After successfully transmitted, the packet is removed from the buffer and the mobile station serves the next packet if exists. The retransmission process is repeated until the packet is successfully received.

In order to analyze the relationship between the transmission probability and the system performance, 
simulations were carried out in terms of number of simultaneously transmitted packets, throughput, and average delay versus the transmission probability for various values of offered load. The average delay is defined as the average time between the arrival of packet in the buffer and its successful reception at the base station. Simulation results are depicted from Fig.4 to Fig.6, where $G$ stands for the offered load. It is assumed that the packet length is 432 bits, the processing gain is $64, E_{b} / N_{0}$ is $15 \mathrm{~dB}, K$ is 100 , and the transmission probabilities in the contention and retransmission state are set equal to $P$. The offered load is defined as the average number of packets arriving in the system during one slot time.

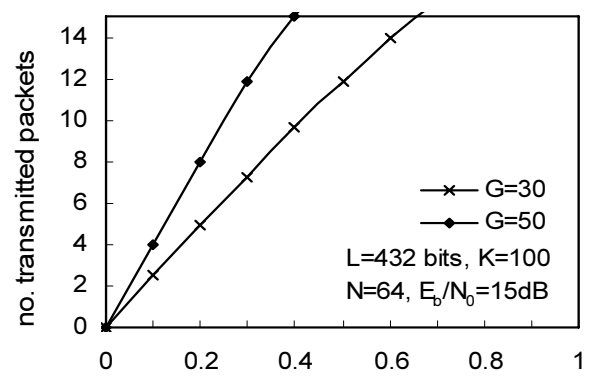

Transmission probability, $\mathrm{P}$

Fig.4. Number of transmitted packets versus transmission probability.

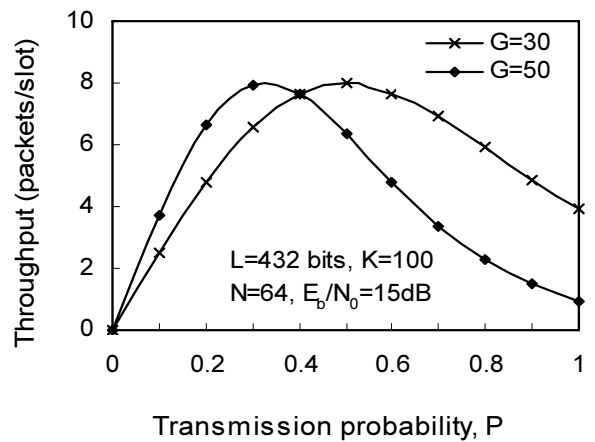

Fig.5. Throughput vs. transmission probability.

As shown in Fig.4, the number of simultaneously transmitted packets monotonously increases with the transmission probability and offered load increasing. From Fig.5, the maximum throughput for the offered loads $G=30$ and $G=50$ can be achieved when $P=0.5$ and $P=0.3$, respectively. In these cases, the number of simultaneously transmitted packets is about 12 from Fig.4. This is same as the number of simultaneously transmitted packets for achieving the maximum throughput as shown in Fig.1. The average delay for the offered loads $G=30$ and $G=50$ increases while decreasing the transmission probability when $P<0.5$ and $P<0.3$, respectively. This is because the mobile stations excessively restrict their transmissions. Furthermore, at a high offered load $(G=50)$, packet errors will frequently occur due to the increased multiple access interferences as the transmission probability increases. Therefore, almost all the mobile stations should retransmit their packets, and hence the average delay increases rapidly.

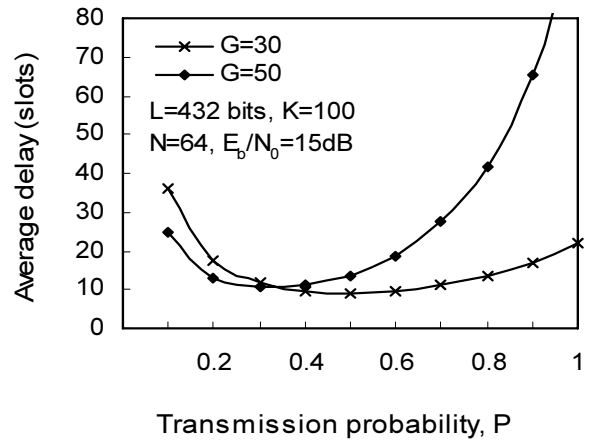

Fig.6. Average delay vs. transmission probability.

\section{PROPORTIONAL BACKOFF SCHEME}

The system model for the proposed scheme, which is named as the Proportional Backoff (PB) scheme, is presented in Fig.7. In the proposed scheme, the base station controls the transmission probability of mobile stations in the centralized manner. The mobile stations in the contention state and retransmission state attempt to transmit packets with the transmission probability $P_{n}$ and retransmission probability $P_{r}$, respectively. The base station calculates these probabilities based on the estimated traffic load and broadcasts over an error-free downlink control channel.

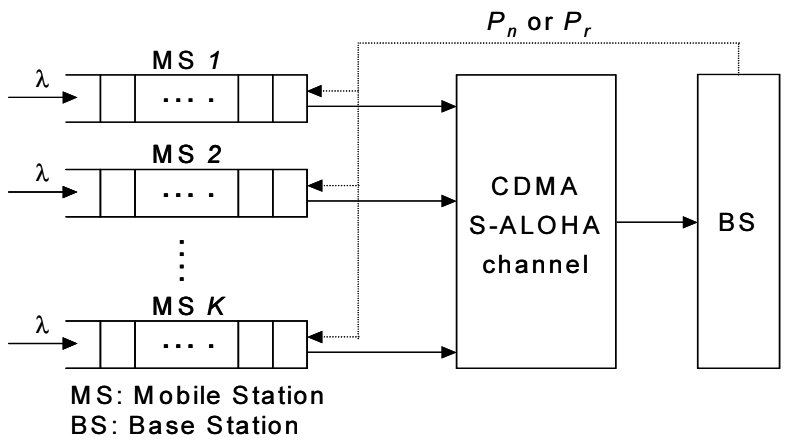

Fig.7. System model of backoff control scheme.

The mobile station that fails to transmit its packet at slot $\mathrm{t}$ retransmit with $P_{r}(t+1)$ at slot $(t+1)$, while the mobile station that enters into the contention state at slot $t$ transmit with $P_{n}(t+1)$ at subsequent slot. The $P_{n}(t+1)$ and $P_{r}(t+1)$ are calculated as follows:

$$
P_{n}(t+1)= \begin{cases}1, & \text { if } N_{r}(t+1) \leq T H_{m} \\ 0, & \text { otherwise }\end{cases}
$$




$$
P_{r}(t+1)= \begin{cases}1, & \text { if } N_{r}(t+1) \leq T H_{m} \\ \frac{T H_{m}}{N_{r}(t+1)}, & \text { otherwise }\end{cases}
$$

where $T H_{m}$ is the number of simultaneously transmitted packets at which the system throughput can be maximized, and $N_{r}(t+1)$ is the number of mobile stations in the retransmission state at slot $(t+1)$. Let $N_{f}(t)$ be the number of unsuccessful mobile stations at slot $t$, and $N_{b}(t)$ the number of mobile stations not permitted at slot $t$. Then $N_{r}(t+1)$ can be derived as follows:

$$
N_{r}(t+1)=N_{f}(t)+N_{b}(t)
$$

The value $T H_{m}$ can be determined by (4), and $N_{b}(t)$ is given by

$$
N_{b}(t)=N_{n}(t-1)\left\{1-P_{n}(t)\right\}+N_{r}(t)\left\{1-P_{r}(t)\right\}
$$

$N_{n}(t)$ in (8) is the number of mobile stations that enter into the contention state at slot $t$, and can be derived by

$$
N_{n}(t)=\left\{K-N_{r}(t)-N_{n}(t-1)\right\} \cdot \lambda
$$

In (9), $K$ is the number of registered mobile stations in the system, and $\lambda$ is the probability that a mobile station generates a packet in each slot. The base station cannot exactly know how many packets are generated in one slot. Therefore, $\lambda$ is computed using a moving time average number of new packets that are successfully received.

In the proposed scheme, if the number of mobile stations in the retransmission state is less than $T H_{m}$, all the mobile stations in both the contention state and the retransmission state should be allowed to transmit a packet. If the number of mobile stations in the retransmission state becomes more than $\mathrm{TH}_{m}$, the base station sets $P_{n}$ into 0 to suppress the transmission of new packets. Also, in this case, the base station sets $P_{r}$ as the values at which the total number of simultaneously retransmitted packets becomes $T H_{m}$, in order to minimize the transmission delay.

\section{SIMULATION RESULTS}

This section presents simulation results for the proposed scheme. For simulation, it is assumed that the packet length is 432 bits equal to a slot duration, the processing gain is $64, E_{b} / N_{0}$ is $15 \mathrm{~dB}$, the total number of mobile stations is 100 , and each mobile station generates packets according to the Poisson process. With theses parameters, it can be seen that $T H_{m}$ is equal to 12 from Fig.1. Also, it is assumed that the length of window used for computing the moving time average of packet arrival rate is set to 1,000 slots.
The performance measures of interest are the system throughput, average delay, and throughput fairness index. The system throughput is defined as the number of successfully transmitted packets during one slot time. The average delay is defined as the average time between the arrival of packet and its successful reception at the base station. And the throughput fairness index is defined as follows [15]:

$$
\text { Fairness }=\frac{\left(\sum_{i=1}^{K} Y_{i}\right)^{2}}{K \sum_{i=1}^{K} Y_{i}^{2}}
$$

where $Y_{i}$ is the measured throughput for the mobile station $i$, and $K$ is the total number of mobile stations in the system.

In this paper, the performance of the proposed PB scheme is evaluated and compared with the existing Harmonic Backoff (HB) scheme [11][12]. In the HB scheme, the mobile station that fails in transmission decreases its transmission probability independently with the traffic load. For the first attempt of packet transmission, the transmission probability $P_{1}$ is set to 1 . If the transmission becomes unsuccessful, then $P_{i+1}$ for $(i+1)$ th attempt is decreased according to

$$
\begin{aligned}
& P_{i+1}=\frac{1}{P_{i}+1}, i \geq 1 \\
& P_{1}=1
\end{aligned}
$$

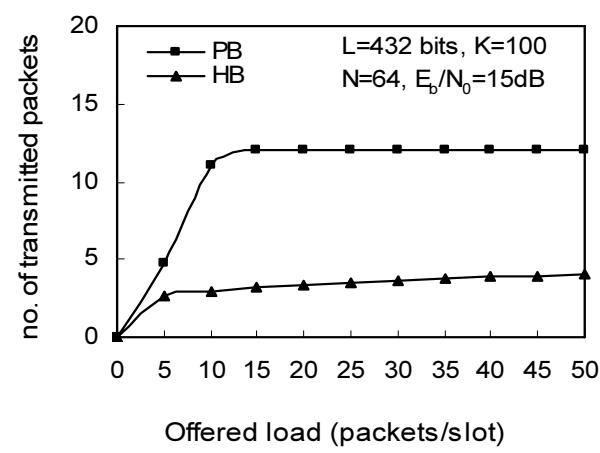

Fig.8. Number of transmitted packets vs. offered load.

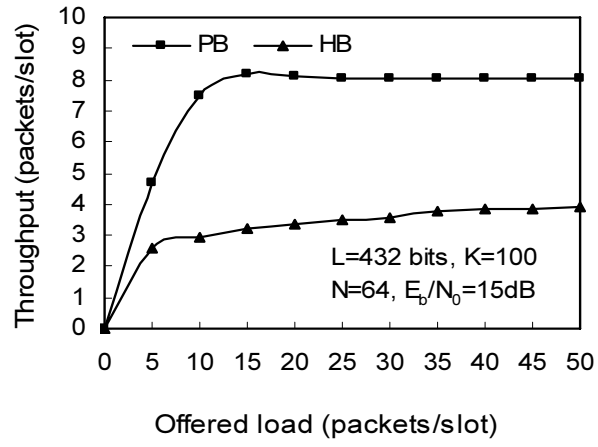

Fig.9. Throughput vs. offered load. 


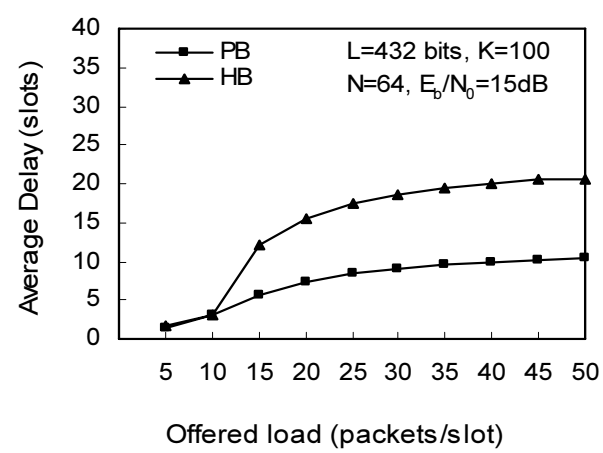

Fig.10. Average delay vs offered load.

The number of simultaneously transmitted packets, throughput, and average delay versus the offered load are shown in Fig.8, Fig.9, and Fig.10, respectively. In the HB scheme, as the traffic load increases, so the bit error rate of each packet increases because of the unconditional transmission of new packets. Therefore, the mobile station in the retransmission state continuously decreases the transmission probability due to the packet error, and moreover, the transmission probability of a specific mobile station becomes excessively decreased. As a result, as shown in Fig.8, the number of simultaneously transmitted packets in the HB scheme is far less than $T H_{m}$. On the other hand, in the proposed PB scheme, the base station controls the transmission probability based on the traffic load and the threshold $T H_{m}$. Therefore, PB scheme keeps the number of simultaneously transmitted packets in the threshold $T H_{m}$ in spite of the increased offered load.

From Fig.9 and Fig.10, PB scheme gives better performance than the HB scheme. The reasons are as follows: i) $\mathrm{PB}$ scheme can control the number of simultaneously transmitted packets more precisely than HB scheme; ii) in the PB scheme, the base station does not permit the transmission of new packets in case of the heavy traffic load.

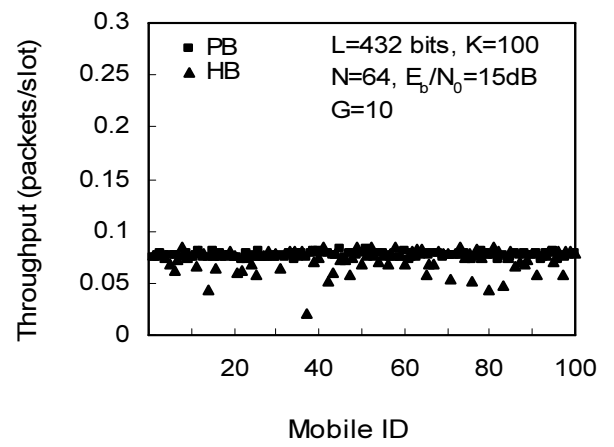

Fig.11. Throughput of each mobile station $(G=10)$.

Fig.11 and 12 shows the throughput of each mobile station when the offered load $(G)$ is 10 and 50 , respectively. With a light traffic load i.e. $G=10$, there is no significant difference in the throughput of each mobile station. As shown in Fig.11, both schemes provide a good fairness when the offered load is low. In the HB scheme, the mobile stations in the retransmission state continuously decrease the transmission probability, while the mobile stations in the contention state transmit their packet with the probability $P_{1}$. Therefore, the mobile stations in the contention state are permitted to transmit a packet prior to the mobile stations in the retransmission state. On the other hand, in the PB scheme, when the number of mobile stations in the retransmission state is more than $T_{m}$, the mobile stations in the contention state are not permitted to transmit a packet. Therefore, as shown in Fig.12, the proposed PB scheme provides fairer throughput for each mobile station than the HB scheme when the offered load is high.

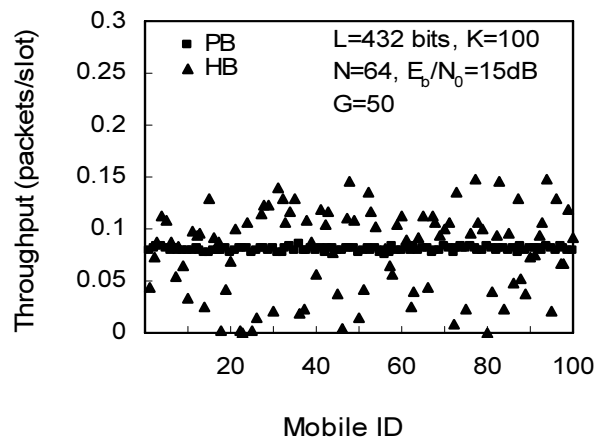

Fig.12. Throughput of each mobile station $(G=50)$.

Fig.13 compares the fairness index according to the offered load. The fairness index of HB scheme decreases sharply when the offered load increases. However, the proposed $\mathrm{PB}$ scheme maintains the fairness index into almost 1 regardless of the offered load. Therefore, PB scheme can guarantee fair packet transmissions among all the mobile stations even though the offered load increases.

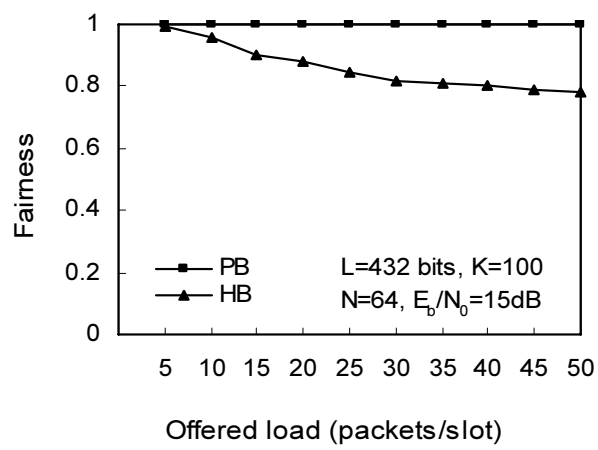

Fig.13. Fairness index.

\section{CONCLUSIONS}

This paper has proposed a centralized backoff control scheme for S-ALOHA CDMA system with a transmitterbased code method. In the proposed scheme, the base station determines the packet transmission probability of 
mobile stations according to the offered load and then broadcasts to all the mobile stations. Simulation results show that the proposed scheme maintains the level of MAI at the optimum threshold. The throughput and delay performance of the proposed scheme outperforms by far those obtained with the existing scheme. In particular, the proposed scheme can guarantee the fair throughput among all the mobile stations.

\section{REFERENCES}

[1] H. Jiang, W. Zhuang, X. Shen, and Q. Bi, "Quality-of-service provisioning and efficient resource utilization in CDMA cellular communications," IEEE Journal on Selected Areas in Commun., vol. 24, no. 1, pp. 4-15, Jan. 2006.

[2] I. Bergel, and H. Messer, "Optimization of CDMA systems with respect to transmission probability, part I: Mutual information rate optimization," IEEE Trans. on Wireless Commun., vol. 7, no. 6, pp. 2075-2083, june 2008.

[3] J. W. So, I. Han, B. C. Shin, and D. H. Cho, "Performance analysis of DS/SSMA unslotted ALOHA system with variable length data traffic," IEEE Journal on Select. Areas Commun., vol.19, no.11, pp.2215-2224, Nov. 2001.

[4] TIA TR45.5, The CDMA2000 RTT candidate submission to ITU-R, Draft Document, June 1998.

[5] B. H. Walke, Mobile Radio Networks: Networking and Protocols, John Wiley \& Sons, 1999, pp.88-108.

[6] D. Makrakis, and K. M. Sundaru Murthy, "Spread slotted ALOHA techniques for mobile and personal satellite communication systems," IEEE J. Select. Areas Commun., vol.10, no.6, Aug. 1992, pp.985-1002.

[7] M. Saquib, S. Das, G. Mandyam, and M. Z. Win, "Fade-resistant CDMA transmission and reception over time-varying wireless channels," IEEE Trans. Wireless Commun., vol. 5, no. 5, pp. 1197 1206, May 2006.

[8] E. S. Sousa, and J. A. Silvester, "Spreading code protocols for distributed spread-spectrum packet radio networks," IEEE Trans. Commun., vol.36, no.3, Mar. 1988, pp.272-280.

[9] O. Sallent, and R. Agusti, "A proposal for an adaptive S-ALOHA access system for a mobile CDMA environment," IEEE Trans. Veh. Tech., vol.47, no.3, Aug. 1998, pp.977-986.

[10] H. Jiang, W. Zhuang, X. Shen, and Q. Bi, "Quality-of-service provisioning and efficient resource utilization in CDMA cellular communications," IEEE J. on Selected Areas in Commun., vol. 24, no. 1, pp. 4-15, Jan. 2006.

[11] S. Choi, and K. G. Shin, "An uplink CDMA system architecture with diverse QoS guarantees for heterogeneous traffic," IEEE/ACM Trans. Networking, vol.17, no.5, Oct. 1999, pp.616628.

[12] Ch. V. Verikoukis, and J. J. Olimos, "Up-link performance of the DQRUMA MAC protocol in a realistic indoor environment for WATM networks," Proc. of VTC2000, Sept. 2000, pp.1650-1655.

[13] TIA/EIA/IS-95-A, "Mobile station-base station compatibility standard for dual-mode wideband spread spectrum cellular systems," Telecommun. Indust. Assn., May 1995.

[14] J. F. Frigon, and V. C. M. Leung, "A pseudo-Bayesian ALOHA algorithm with mixed priorities," ACM Wireless Networks, vol.7, Issue 1, Jan. 2001, pp.55-63.

[15] R. Jain, D. M. Chiu, and W. Hawe, "A Quantitative Measure of Fairness and Discrimination for Resource Allocation in Shared Systems," DEC Research Report TR-301, 1984.

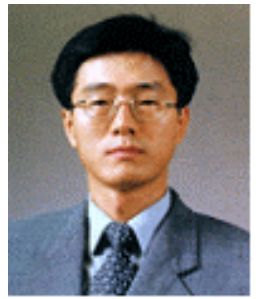

Intaek Lim received the B.S. degree in computer science from University of Ulsan, Ulsan, Korea, in 1984, and the M.S. degree in computer science and statistics from Seoul National University, Seoul, Korea, in 1986. He received the $\mathrm{Ph}$. D. degree in computer engineering from University of Ulsan, Ulsan, Korea, in 1998. From 1986 to 1993, he was a Senior Researcher at Samsung Electronics Co., Ltd. In 1998, he joined the faculty at Pusan University of Foreign Studies, Busan, Korea, where he is currently a Professor in the Department of Embedded IT. From July 2006 to June 2007, He was a visiting scholar at Cleveland State University in USA. His research interests include the MAC protocol design, ad-hoc network, RFID, and mobile computing. 